\title{
Operative treatment of aneurysms and Coanda effect: a working hypothesis ${ }^{1}$
}

\author{
J. L. ROBINSON AND A. ROBERTS \\ From the Neurosurgical Centre, Liverpool, and the Liverpool College of Technology
}

SUMmARY The Coanda effect has been known to mechanical engineers for many decades. Consideration of this effect both by a neurosurgeon and a mechanical engineer revealed that it might be of importance during the operative treatment of intracranial aneurysms. If a jet effect were produced in the stream of blood after clipping an aneurysm, most of the flow of blood might be directed down only one limb of a bifurcation occurring in a vessel near to a clipped aneurysm. Blood might also be entrained from the other limb of the bifurcation. This boundary wall effect, which can occur without the rate of flow through the vessel being altered appreciably, could explain some of the unfortunate sequelae of aneurysm surgery which occur in the absence of any obvious cause such as postoperative thrombosis, etc. A possible mechanism for some of the complications after gradual occlusion of the common carotid artery in the neck is also proposed on this basis. Other details of how this data might be of clinical significance, together with suggestions for how to avoid fluidic effects during aneurysm surgery, are presented.

There are many untoward results that may overtake a vessel which has been both the seat of an aneurysm and the site of an operative procedure. These happenings are usually associated with an unfortunate outcome for the patient. Although many causes for morbidity and mortality during or after aneurysm surgery are known-for example, rupture of aneurysm during operation, spasm, thrombosis, etc.- at least on some occasions, post mortem examination of the operation site reveals nothing untoward. This paper is a short analysis of some fluid mechanic effects that might help to explain an unsatisfactory result to an uneventful operation.

\section{BACKGROUND}

When a jet of fluid enters a wide vessel containing a similar fluid, it entrains the fluid and becomes broader as it moves downstream. The removal of fluid from the liquid surrounding the jet lowers the pressure between the jet and the side walls of the container. The low pressure zone that is produced induces a backward flow from the down-

1 This work was carried out during the tenure of a grant from the Wellcome Trust. stream area which is now at a relatively higl pressure. If a disturbance causes the jet to move towards one wall, then the upstream area foB replenishing the flow is reduced, and the counter flow is reduced. The opposite effect occurs on the other side of the jet, with the result that the jet is deflected still further until it is forced completely against one wall. Conditions are now stable and the jet remains attached to the wall. This is known as the Coanda or boundary wall effect (American Society of Mechanical Engineers, 1962; Kirshner, 1966).

Figure 1 shows a diagram of a symmetrical constriction in a ' $\mathrm{Y}$ ' shaped tube system illustrating the normal flow of fluid and Fig. 2 shows how, after the jet has been deflected by signal ' $S$ ', virtually all of the flow is deflected into the left hand limb of the ' $Y$ ' piece.

The limits for production of these conditions are reasonably wide. The angle $\theta$ in Figs 1 and 2 has been drawn rather small so that the diagrams are as clear as possible, but it could certainly be as much as $140^{\circ}$ or possibly more, without altering this fluidic effect. For values of $\theta$ much above $140^{\circ}$, a boundary wall effect will be produced 


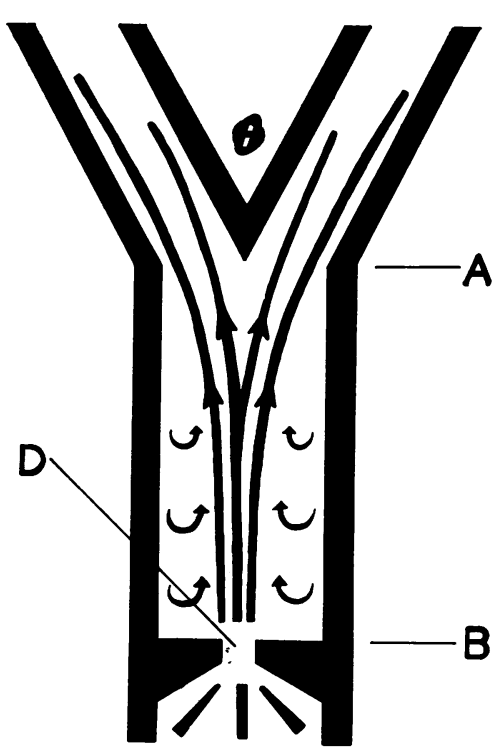

FIG. 1. Diagram of fluid flow in a Y-shaped tube when there is a symmetrical constriction in the main limb of the ' $Y$ '. $\mathrm{A}-\mathrm{B}=$ distance from jet to splitter. $\mathrm{D}=$ diameter of jet. $\theta=$ angle of arms of ' $Y$ '.

only if the constriction is asymmetrical and situated very close to the splitter.

The distance between the jet and the splitter (AB in Fig. 1) can also be varied. The greater the distance $A B$, the more marked will be the fluidic effect up to a limit when $A B$ is of the order of 10 times the diameter of the jet ( $D$ in Fig. 1). Values for $\mathrm{AB}$ above this will give progressively less effect. In the range of lower distances between jet and splitter, the Coanda effect will be very slight, but in the higher range, the flow becomes more and more bistable, and the signal ' $S$ ' in Fig. 2 will still be effective at as low a pressure as $0.002 \mathrm{~mm} \mathrm{Hg}$ and a very marked Coanda effect would still be produced. Coanda effect has been observed for Reynold's numbers as low as 50 - that is, the effect is observed for both laminar and turbulent flow, and will occur in elastic as well as rigid tube systems.

The channelling effect of the jet down one limb only of the ' $\mathrm{Y}$ ' piece will, of course, only occur provided that the diameter of the jet at that level is less than the diameter of the limb of the ' $Y$ '.

\section{APPLICATION}

During clipping of an aneurysm one aims to exclude the aneurysm from the circulation without in any way interfering with, or distorting, adjacent blood vessels and their divisions. A perfectly placed clip would cause no problem, but there are situations - for example, clipping of a sessile aneurysm-in which distortion of the parent vessel is a real possibility.

Direct operative attacks on aneurysms are frequently carried out under hypotension, and this usually of sufficient degree to cause a corresponding decrease in the diameter of the intracranial blood vessels. Postoperatively, as the blood pressure returns to normal and the vessels dilate up to their normal size, a clip may be found to have caused an encroachment on the lumen of the parent vessel which was not obvious at the time of operation. It may be particularly difficult to

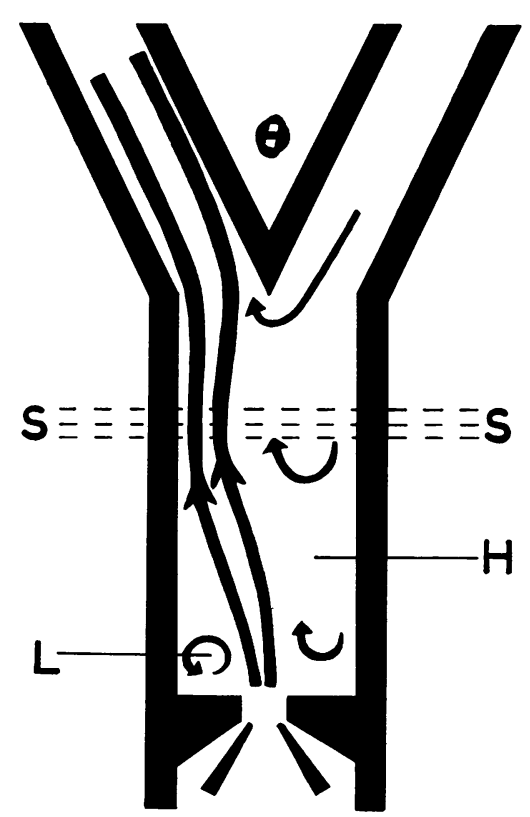

FIG. 2. Diagram showing how once a jet has been deflected by a signal, the high and low pressure areas will keep it stably applied to the wall of the vessel even if the signal is discontinued. $\mathrm{H}=$ area of high pressure. $\mathrm{L}=$ area of low pressure, or separation bubble. $\mathbf{S}=$ signal. $\theta=$ angle of arms of ' $Y$ ', which can be at least as great as $140^{\circ}$. 
assess potential distortion under hypotensive conditions if a broad-necked aneurysm has had to be clipped parallel to the parent vessel, as the aneurysm will sustain a relatively large decrease in size, as compared with the parent artery, when the blood pressure drops.

If the internal dimension of the neck of an aneurysm is less than one and a half times the diameter of the parent vessel, and if a clip is placed too tightly at the base of an aneurysm so that encroachment upon the lumen of the parent vessel does occur, then the situation is markedly different, according to whether the clip be placed at right angles or parallel to the parent vessel. In the former case, the tension in the vessel wall from the clip will be very localized and will therefore cause the vessel to take a curve, with the concavity of the curve on the side of the vessel bearing the clip. This curve, however, will be of such a degree that it ensures that the diameter of the vessel is unaffected. In the latter case, however, the tension will be distributed along the length of the vessel just a little greater than that of the clip, and there will be no position that the vessel can take up which will nullify the effect of this constriction.

Since the velocity of flow through a vessel is proportional to the square of the diameter of the vessel, a comparatively small constriction can produce quite a marked jet effect. Furthermore, if the parent vessel is distorted during clipping of an aneurysm, then the constriction of the vessel will not be symmetrical. On the contrary, as the clip with the narrowed segment of vessel will be on one side of the vessel only, a jet will be produced which will already be stably applied to the opposite wall of the parent vessel. In other words, the signal ' $S$ ' in Fig. 2 is not necessary for the production of the boundary wall effect if the constriction causing the jet is asymmetrical. By this means, a postoperative Coanda effect might occur after aneurysm surgery. If this did happen, it would divert the flow of blood almost entirely down only one limb of a bifurcation occurring near an aneurysm that had been clipped parallel to the parent vessel in such a way as to distort that vessel. To make matters worse, blood could be entrained from the other limb of the bifurcation causing retrograde flow in that vessel, thus increasing the risk of ischaemic damage to the area of brain supplied by that vessel. All these unfortunate effects could occur with a constriction not sufficiently marked to alter the flow rate through the vessel in question

\section{CONSEQUENCES}

Although there is no direct evidence that the Coanda effect has occurred clinically, we felt there was a strong case to be made for avoiding. even the possibility of this preventable and po- $\overrightarrow{\vec{F}}$ tentially lethal complication of aneurysm surgery. We have therefore drawn up some guide lines which should be of use in avoiding the fluidic effects during the surgical treatment of the commoner aneurysms.

1. INTERNAL CAROTID ARTERY The junction here is roughly $\mathrm{T}$-shaped, the angle $\theta$ approximating to $180^{\circ}$. If a normal clip were used an aneurysm would be clipped at right angles to the parent vessel and even if the clip were placed too close to the internal carotid artery, curvature of that vessel would prevent any jet effect occurring However, if a Teflon Sundt-Kees clip (Dow $\overline{\mathbf{Q}}+\bar{\phi}$ Brothers, Mitcham, Surrey, England) were usec을 there would be a possibility of a constriction of $\vec{B}$ the area as this clip lies parallel to the interna carotid artery. As can be seen (Fig. 3) the cong striction would have to be very high on the inte? nal carotid artery for a boundary wall effect te.

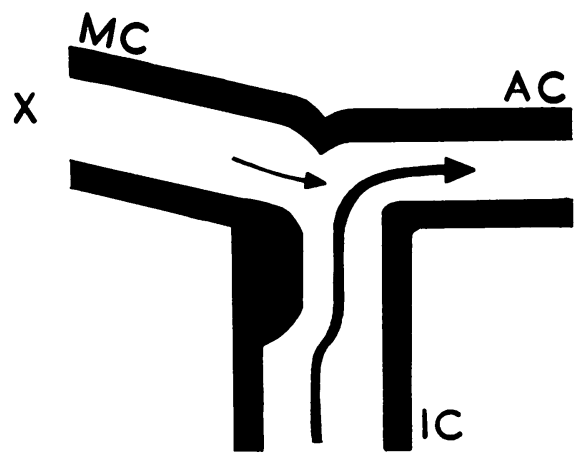

FIG. 3. Clip applied to high aneurysm of internal carotid artery. The normally satisfactory longitudinal Sundt-Kees clip is here potentially dangerous, causing diversion of the blood stream down the anterior cerebral artery. $\mathrm{AC}=$ anterior cerebral artery. $\mathrm{IC}=$ internal carotid artery. $\mathrm{MC}=$ middle cerebral artery. $\mathrm{X}=$ ischaemic territory. 

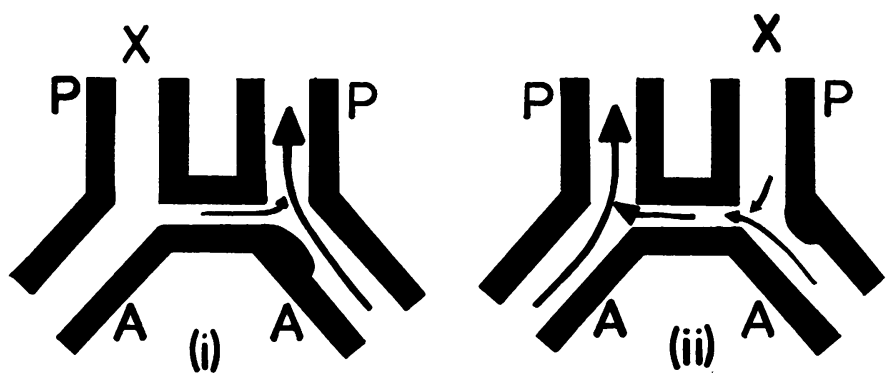

FIG. 4. Anterior communicating artery complex. (i) Medial, inferomedial, or superomedial distortion will cause entrainment of blood from opposite pericallosal. (ii) Lateral, inferolateral, or superolateral distortion will cause diversion of jet across the anterior communicating artery and up to the contralateral pericallosal artery. Blood will be entrained from the ipsilateral

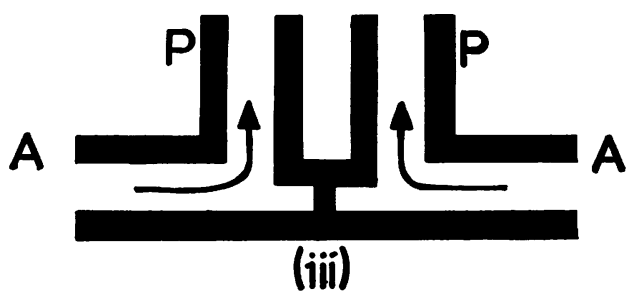
pericallosal. (iii) Constriction or obliteration of the anterior communicating artery itself precludes the possibility of a Coanda effect. $\mathrm{A}=$ anterior cerebral artery. $\mathbf{P}=$ Pericallosal artery. $\mathrm{X}=$ area of ischaemia.

occur because of the shape of the bifurcation. Lower aneurysms could clearly be clipped by any method without risking this, but if an aneurysm were high on the internal carotid artery, then it would be important to clip this at right angles to the internal carotid artery. One of us (J. R.) has seen two patients in whom a Sundt-Kees clip was applied to a high carotid aneurysm and in whom the postoperative result was unsatisfactory. Both patients had smooth surgical interventions with satisfactory clipping of the aneurysm. Both patients had marked postoperative hemipareses. Postoperative angiography showed no occlusion of a major vessel and the perforating vessels were satisfactorily filled in both cases. The terminal portion of the internal carotid artery was clearly narrowed near its bifurcation, but not sufficiently for it to be expected that the total blood flow would be reduced by this. There was greatly reduced flow of contrast medium into the middle cerebral artery which appeared to be of normal calibre.

2. ANTERIOR COMMUNICATING COMPLEX Aneurysms on the anterior cerebral artery should clearly be clipped at right angles to that vessel if possible.

Figure 4 shows two possible situations when an anterior communicating aneurysm has been clipped nearly parallel to the anterior cerebral artery. Either fluid may be entrained from the opposite pericallosal artery, or the jet may be directed across the anterior communicating artery to the contralateral pericallosal artery. Both these situations would cause ischaemia of the appropriate area-that is, the territory supplied by the pericallosal artery on either sidegrossly out of proportion to the comparatively minor narrowing of the parent vessel caused by the clip. In order to avoid this effect, an aneurysm should ideally be clipped in a position half way between right angles to the anterior cerebral artery and right angles to the anterior communicating artery. If this perfect result cannot be achieved, the clip should be so placed that any constriction produced would affect the anterior communicating artery itself, as obliteration of this artery obviates any fluidic effect occurring in this region.

3. MIDDLE CEREBRAL ARTERY In the case of an aneurysm occurring at the trifurcation of the middle cerebral artery, Coanda effect would not occur. However, in the case of an aneurysm occurring proximal to the trifurcation, it would be important to apply the clip at right angles to the parent vessel. In the event of there being an early bifurcation of the middle cerebral artery with subsequent secondary bifurcation of the greater branch, and an aneurysm occurring on 
the proximal bifurcation, it can be seen (Fig. 5) that it would be a mistake to apply the clip at right angles to the first lesser branch.

4. BASILAR Aneurysm at the tip of the basilar artery would not normally be subject to this effect unless very great distortion at the base of the aneurysm were produced. However, an aneurysm of the basilar trunk would produce an

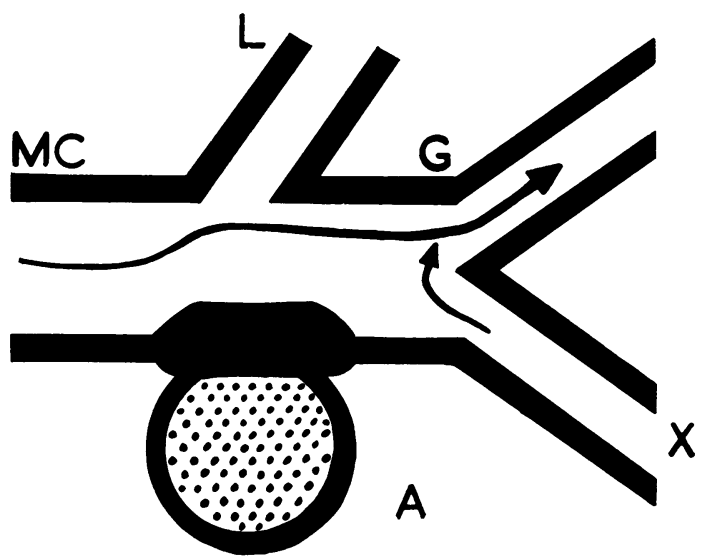

FIG. 5. Early bifurcation with subsequent further bifurcation of larger branch. This diagram shows the possible ill-effects of clipping the aneurysm at right angles to the lesser branch. $\mathrm{A}=$ aneurysm. $\mathrm{G}=$ greater branch, $\mathrm{L}=$ lesser branch. $\mathrm{MC}=$ middle cerebral artery. $\mathrm{X}=$ area of ischaemia.

almost textbook situation for this effect to occur. Theoretically, the constriction causing the jet could be as far as $2 \frac{1}{2} \mathrm{~cm}$ below the bifurcation of the basilar artery into the posterior cerebral arteries.

5. GRADUAL OCCLUSION OF COMMON CAROTID ARTERY IN NECK It is always difficult to be sure what happens during gradual clamping of the common carotid artery in the neck. Particularly confusing is the way that neurological defect may occur at any time, not just within a short time of when the clamp is tightened. The way that neurological signs can disappear after opening of the clamp is also interesting (Tindall and Odom, 1969).
In fact, the clamp would produce a symmetrical constriction giving a central jet. However, if a deflecting signal (' $\mathrm{S}$ ' in Fig. 2) were applied, the Coanda effect could be produced if the constriction of the common carotid artery were within about $6 \mathrm{~cm}$ of its bifurcation into the internal and external arteries. However, a deflecting signal could be produced by moving the clamp, and as the clamp passes through the muscles before emerging through the skin, there will be many things-for example, swallowing, changing the dressing, etc.- that by physically rotating the clamp would produce the necessary deflecting signal! Once the jet was deflected, the boundary wall effect would be stable so the jet would remain applied to the wall, even though the signal had been discontinued. It would, of $\overrightarrow{0}$ course, be a matter of luck whether the jet of blood, stably applied to the wall of the common carotid artery, passed on up the internal or external carotid artery; in the case of the latter, a hemiparesis or hemiplegia, occurring at an unexpected time, would be explained. Blood would $\sigma$ also be entrained from the other branch of theूㅇㅇㅇ common carotid artery, so there would be nњ stasis giving rise to thrombosis either there of just beside the jet where the separation bubble (' $L$ ' in Fig. 2) would be moving round and round. In such circumstances, of course, re moval of the clamp would immediately restore the status quo. In other words, in the absence of thrombosis (prevented by these movements of blood) releasing the clamps would abolish the Coanda effect and allow blood flow to occur normally again and if this were accomplished quickly enough to prevent irreversible ischaemia, then any neurological deficit should disappear.

\section{FURTHER DISCUSSION}

Two further points should be mentioned. First is the effect of intimal cushions on a deflected jet. These intimal cushions are commonly found at bifurcations in the circle of Willis, and are especially prevalent in patients with aneurysms (Hassler, 1961). They occur, however, on the of proximal carina, and are situated at the base of the branch rather than the end of the stem. In $\frac{\text { o }}{2}$ other words, they would not be expected to cause $\frac{D}{0}$ a jet of fluid that was applied to one wall of the stem to deflect back to the centre. On the other $\mathbb{N}$ 
hand, these cushions are usually more than $1 \mathrm{~mm}$ thick, thus they might reduce the effective diameter of the branch so that the diameter of the jet at that point would exceed the diameter of the branch, and some spill over of blood would occur into the other limb of the bifurcation.

The second point concerns the relative weakness of the intracerebral vessel walls. The low pressure area beside the jet might tend to collapse these vessels, thus increasing the effective length of the stenosis, and abolishing the Coanda effect. In practice, this decreased side pressure tending to suck the vessel walls in would be more than balanced by the internal pressure tending to blow them out. An episode of postoperative hypotension, would of course be expected to abolish the effect.

\section{CONCLUSIONS}

Joint consideration of the Coanda effect between a neurological surgeon and a mechanical engineer has suggested that this might be one cause of unfortunate operative results in aneurysm surgery. The Coanda effect might occur as a result of distorting the parent vessel during clipping of an aneurysm and the consequence of this effect would be very marked even if the constriction of the main blood vessel caused by the clip were not enough to alter the flow rate through the vessel. Although much of this material presented is theoretical, in the case of high internal carotid artery aneurysms directly attacked, and slow occlusion by clamp of the common carotid artery in the neck, there is some clinical evidence that this effect might be significant. Be that as it may, the methods for applying clips in various situations to avoid this effect have been worked out and it should often be relatively easy to apply clips in this manner, thus avoiding any possibility of producing a fluidic effect.

\section{REFERENCES}

American Society of Mechanical Engineers (1962). Fluid Jet Control Devices. Papers presented at the Annual Meeting of the Society, New York, 28 November 1962. Edited by F. T. Brown.

Kirschner, J. M. (Ed.) (1966). Fluid Amplifiers. McGraw Hill: New York.

Hassler, O. (1961). Morphological studies on the large cerebral arteries with reference to the aetiology of subarachnoid haemorrhage. Acta Psychiatrica et Neurologica Scandinavica, Suppl. 154, Volume 36.

Tindall, G. T., and Odom, G. L. (1969). Treatment of intracranial aneurysms by proximal carotid ligation. In Progress in Neurological Surgery, Vol. 3, pp. 67-114. Edited by H. Krayenbuhl, P. E. Maspes, and W. H. Sweet. Karger: Basel. 